\title{
Generating comprehensive comparative evidence on various interventions for penile rehabilitation in patients with erectile dysfunction after radical prostatectomy: a systematic review and network meta-analysis
}

\author{
Dechao Feng", Shengzhuo Liü, Yubo Yang, Yunjin Bai, Dengxiong Li, Ping Han, Wuran Wei \\ Department of Urology, Institute of Urology, West China Hospital, Sichuan University, Chengdu, China \\ Contributions: (I) Conception and design: D Feng; (II) Administrative support: P Han, W Wei; (III) Provision of study materials or patients: D Feng, \\ S Liu; (IV) Collection and assembly of data: Y Yang, Y Bai; (V) Data analysis and interpretation: Y Yang, D Li; (VI) Manuscript writing: All authors; \\ (VII) Final approval of manuscript: All authors. \\ \#These authors contributed equally to this work. \\ Correspondence to: Wuran Wei. Department of Urology, Institute of Urology, West China Hospital, Sichuan University, Guoxue Xiang \#37, Chengdu \\ 610041, Sichuan, China. Email: weiwuran@126.com.
}

Background: We aim to present a comprehensive comparison of various treatments in the management of penile recovery after radical prostatectomy $(\mathrm{RP})$ and provide recommendations for future research.

Methods: Literature search of electronic databases including PubMed, the Cochrane Library, Embase, PsycInfo, and Web of Science, and manual retrieval were conducted from inception through March 2020.

"Erectile dysfunction" and "prostatectomy" were used as the Mesh terms. The patients, intervention, comparison, outcome, and study design (PICOS) approach were used to define study eligibility. Two authors independently selected studies, evaluated the methodological quality, and extracted data using Cochrane Collaboration's tools. The data analysis was completed by STATA version 14.2.

Results: A total of 24 studies with 3,500 patients were incorporated in the final analysis after screening 6,131 records. Our findings indicated that vacuum constriction devices (VCD) ranked 1st which meant that patients in VCD group had the best effect regarding mean IIEF scores within 3 months after RP, and no significant difference was observed between VCD and VCD with $20 \mathrm{mg} /$ day tadalafil (V20DT) (MD: 5.44; 95\% CI: -0.81 to 11.69$)$. VCD and $50 \mathrm{mg} /$ day sildenafil (VC50DS) showed superiority over $50 \mathrm{mg} /$ day sildenafil (50DS) (MD: 3.75; 95\% CI: 2.74-4.76) and intraurethral alprostadil 125-250 $\mu \mathrm{g}$ (MD: 3.05; 95\% CI: 0.38 to 5.72 ), respectively. Moreover, V20DT showed significant superiority over the other interventions for $\geq 6$ months mean International Index Erectile of Function (IIEF) scores after RP. Monotherapy appeared to have similar efficacy in terms of mean IIEF scores and proportion of patients return to baseline, and the effect of phosphodiesterase type 5 inhibitors (PDE5is) did not seem to be affected by the patterns of administration (regular or on demand).

Conclusions: The combination therapy showed certain advantages over monotherapy, and we recommended the combination of VCD and PDE5is to be considered in the clinical management of penile rehabilitation after $\mathrm{RP}$.

Keywords: Penile rehabilitation; erectile dysfunction (ED); radical prostatectomy (RP); network meta-analysis

Submitted Apr 29, 2020. Accepted for publication Oct 06, 2020.

doi: 10.21037/tau-20-892

View this article at: http://dx.doi.org/10.21037/tau-20-892 


\section{Introduction}

With the progress of early detection and alleviated health awareness, the morbidity of prostate cancer (PCa) has been growing in the past decades and ranked first among malignancies in men $(1,2)$, among which an increasing number of patients were diagnosed at a younger age $(3,4)$. Though fortunately low is the death rate of PCa, which is reported to be $0.07 \%$ to $0.15 \%$ (5), prostatectomy related erectile dysfunction (ED) is of great concern for its high occurrence and negative effects on quality of life, especially in the sexually active population. With the improved understanding of cavernous nerves (CNs), the pathophysiological basis underlying radical prostatectomy (RP) induced ED was well studied and the most likely mechanism could be the pathophysiological change of cavernous smooth muscle and tunica albuginea induced by CNs injury in operation (6-8). According to previous studies, the prevalence of RP induced ED was about $55.6 \%$ after nerve-sparing laparoscopic radical prostatectomy (nsLRP) and 39.8\% after robot-assisted radical prostatectomy (RARP) (9-12). Though supported by current evidence that nerve-sparing robot-assisted radical prostatectomy (nsRARP) could be the most favorable operation for preserving postoperative erectile function (EF) in PCa patients, the postoperative ED prevalence could still be as high as $10-46 \%$ (13) and most postoperative patients are unlikely to recover to their baseline EF (14). Concerned the current situation that the mean age patients were when diagnosed with PCa has decreased and sexual activityrelated life quality expectancy has grown (15), it is of great importance to establish an effective treatment protocol for postoperative penile rehabilitation. At present, treatment strategies for ED mainly include phosphodiesterase type 5 inhibitors (PDE5is), psychotherapy intervention, pelvic floor muscle training (PFMT), intracorporeal injection of a vasoactive substance, vacuum constriction devices (VCD), and penile prosthesis implant. Other therapeutic approaches including stem cell therapy, nerve transplantation, lowintensity extracorporeal shockwave therapy, erythropoietin, tacrolimus, and hyperbaric oxygenation therapy also show promising efficacy for ED management.

However, it should be noted that most of the prostatectomy-related ED could be resulted from $\mathrm{CNs}$ injury and the concept of ED could be categorized into primary impotency and secondary impotency for different pathogenesis. Thus, confusions could be aroused that whether above illustrated therapeutic approaches could be as effective as for RP induced ED and if the guidelines for $\mathrm{ED}(16,17)$ could be ideally adaptive for RP induced ED. From the results of the previous meta-analysis, PDE5is, VCD, PFMT, and intracorporeal injection was effective for penile rehabilitation after RP (18-22). Because most of the RCTs conducted in this context were placebo-controlled designed and there is a lack of data from the direct comparison between therapies. It could be difficult for physicians to determine the most preferable treatment strategies for patients when faced with a large variety of options. To make a synthesis of potentially practicechanging evidence and provide a trustworthy evidence-based reference on postoperative penile rehabilitation therapy for physicians, we performed this network meta-analysis. We present the following article in accordance with the PRISMA reporting checklist (available at http://dx.doi.org/10.21037/ tau-20-892).

\section{Methods}

\section{Study Selection}

In accordance with the statement of preferred reporting items for systematic review and meta-analysis (PRISMA) (23), electronic databases including PubMed, the Cochrane Library, Embase, PsycInfo, and Web of Science were searched to identify RCTs from inception through March 2020 with no limitation to language. "erectile dysfunction" and "prostatectomy" were used as the Mesh terms, and the search strategy used in PubMed was as follows: $((()((()(($ Prostatectomy[Title/ Abstract]) OR Prostatectomies[Title/Abstract]) OR Prostatectomy, Suprapubic[Title/Abstract]) OR Prostatectomies, Suprapubic[Title/Abstract]) OR Suprapubic Prostatectomies[Title/Abstract]) OR Suprapubic Prostatectomy[Title/Abstract]) OR Prostatectomy, Retropubic[Title/Abstract]) OR Prostatectomies, Retropubic[Title/Abstract]) OR Retropubic Prostatectomies[Title/Abstract]) OR Retropubic Prostatectomy[Title/Abstract])) AND $(((()(($ Erectile Dysfunction[Title/Abstract]) OR Dysfunction, Erectile[Title/Abstract]) OR Male Sexual Impotence[Title/ Abstract]) OR Impotence, Male Sexual[Title/Abstract]) OR Sexual Impotence, Male[Title/Abstract]) OR Male Impotence[Title/Abstract]) OR Impotence, Male[Title/ Abstract]) OR Impotence[Title/Abstract]). The details of search strategy were shown in supplementary material. Besides, we also retrieved reference lists of included studies and related reviews to ensure the comprehensive search. 


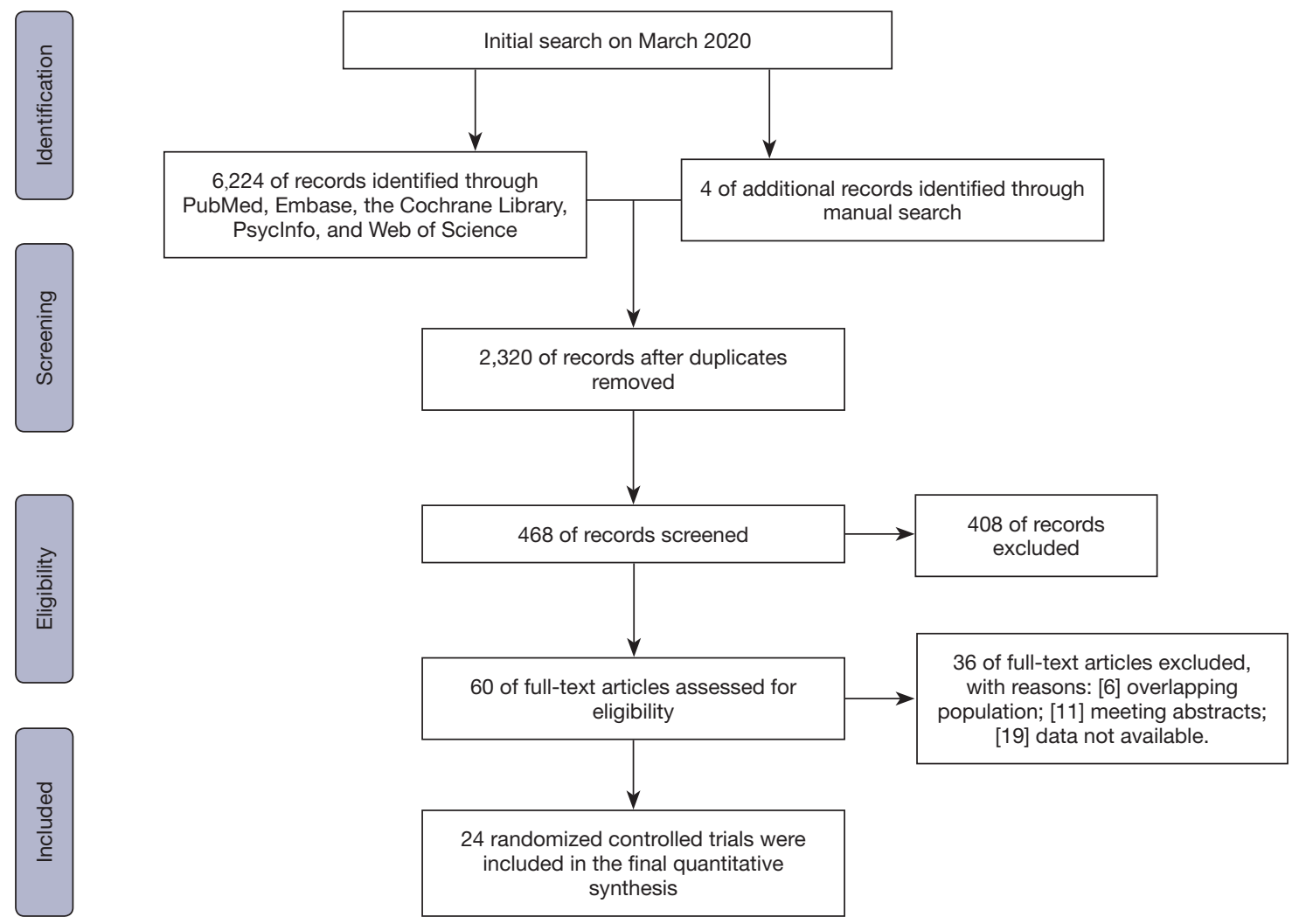

Figure 1 PRISMA flowchart of the study selection process for network meta-analysis.

Four independent reviewers identified potentially eligible studies after removing duplicates and screening titles and abstracts. Full-text articles that met the following inclusion criteria and presented available data were included in the final analysis. Any disagreement was resolved by consensus with a third party. Figure 1 depicts the PRISMA flowchart.

\section{Selection criteria}

The patients, intervention, comparison, outcome, and study design (PICOS) approach was used to define study eligibility; patients $(\mathrm{P})$ : patients with ED after RP; intervention (I): patients treated with any drug, supportive therapy or device; comparison (C): comparisons with different interventions; outcomes $(\mathrm{O})$ : EF measured by International Index Erectile of Function (IIEF); study design (S): randomized controlled trial (RCTs) with sufficient data for extraction were selected. Meeting abstracts, overlapping population, and duplicated studies were excluded.

\section{Quality assessment}

Two independent reviewers (DCF, SZL) evaluated the study quality (Figure 2) according to the Cochrane Collaboration's Risk of Bias (RoB) tool in Review Manager software (https://community.cochrane.org/help/toolsand-software/revman-5). This tool primarily evaluates 7 domains: random sequence generation (selection bias); allocation concealment (selection bias); blinding of participants and personnel (performance bias); blinding of outcome assessment (detection bias); incomplete outcome data (attrition bias); selective reporting (reporting bias); other bias (such as funding sources). Besides, the level of evidence of each included article was rated independently by DCF and SZL using the Oxford Centre for EvidenceBased Medicine criteria (24). This scale graded studies from strongest (level 1) to weakest (level 5) strength of evidence based on study design and data quality. Figure 2 details the RoB summary of the included articles in this study. Overall, included studies had a low risk of random sequence generation (selection bias), incomplete outcome data (attrition bias), and selective reporting (reporting bias). The 


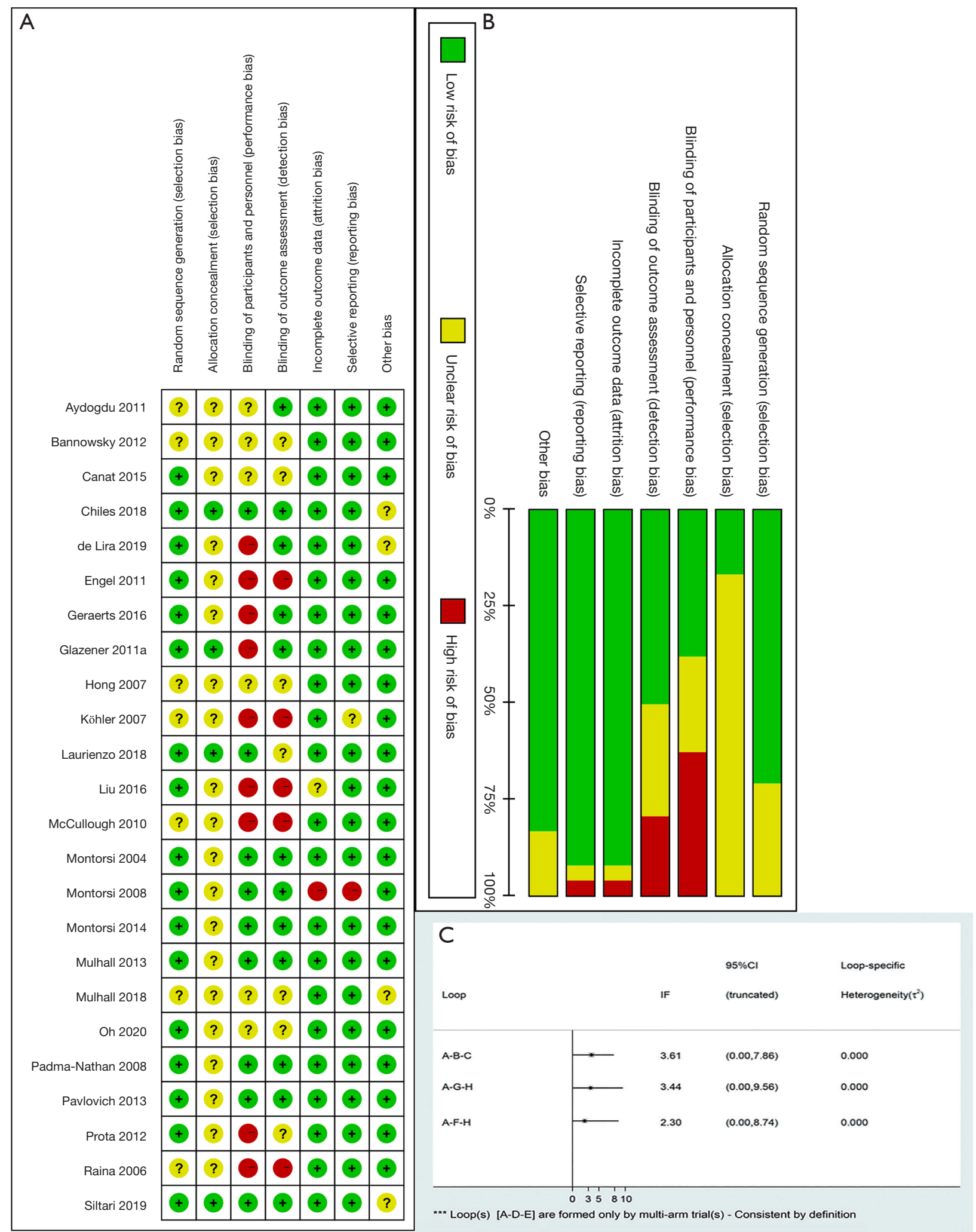

Figure 2 Methodological quality of trials included in the meta-analysis and loop inconsistency. (A) risk of bias summary; (B) risk of bias graph; (C) loop inconsistency. 
risk of allocation concealment was unclear due to the lack of related descriptions. Besides, 19 of 24 RCTs were rated as $1 \mathrm{~b}$ and regarded as high quality (Table 1).

\section{Statistical analysis}

Data presented as mean and standard deviation (SD) were used. Median and range were used to estimate mean and SD (25). The percentiles, 25 th, and 75 th percentiles as well as 5 th and 95th percentiles, were transformed to SD through the following formula: $\mathrm{SD} \approx$ Norm $\mathrm{IQR}=(\mathrm{P} 75-\mathrm{P} 25) \times 0.7413$ (IQR: inter-quartile range, $\mathrm{P} 75: 75$ th percentile, P25: 25 th percentile) (26). Dichotomous data were calculated as risk ratios (RR) and continuous outcomes as mean difference (MD), both with $95 \%$ confidence intervals (CIs). The network meta-analysis was performed based on the Bayesian framework model using STATA version 14.2. The random-effects model was used unless the program suggested no heterogeneity. Besides, we performed loop inconsistency with inconsistency factor (IF) and local inconsistency with node-splitting analysis to evaluate the consistency of indirect and direct comparisons. We identified the source of inconsistency through sensitivity analysis and our results would be relatively robust if we cannot explain the inconsistency. Publication bias was assessed by the symmetry of funnel-plots. We ranked the various interventions based on the values of surface under the curve cumulative probabilities (SUCRA). The statistical significance was defined as $\mathrm{P}<0.05$.

\section{Results}

\section{Search results}

Six thousand and six hundred twenty-eight records were initially identified from the database, and 60 studies were potentially eligible for further review in full text. Of these studies, 36 were excluded on the basis of inclusion criteria, and 24 studies (27-50) were incorporated in the final analysis.

The 24 articles contained 3,500 patients and were published between 2004 and 2019. In total, 3,500 patients were enrolled in these trials and most of the trials were deemed to be high quality according to the Oxford Centre for Evidence-Based Medicine criteria (24). The 24 studies contained seven interventions, 14 involving PDE5is, 6 involving PFMT, 4 involving vacuum therapy, 1 involving neuromodulatory therapy, 2 involving statin therapy, 1 intracorporeal injection therapy, and 1 involving hyperbaric oxygenation therapy. Of these studies, 8 assessed the combined effects of these treatments. The baseline characteristics of the included studies are described in Table 1. Besides, the network plots of investigated outcomes were presented in Figure 3.

\section{Network meta-analysis of mean IIEF scores}

Two parallel-group network meta-analysis were conducted to compare the efficacy of different treatments in terms of short-term results of mean IIEF scores due to available data in the included studies (Figure 4). The first group incorporated 11 studies with 1,235 participants involving 11 interventions (Figure 4E). VCD, VCD and $20 \mathrm{mg} /$ day tadalafil (V20DT), and $200 \mathrm{mg}$ avanafil on demand (200AOD) increased mean IIEF scores within 3 months after RP significantly more than many other treatments (Figure 4E). Most differences between the remaining interventions were small or very uncertain. According to the SUCRA ranking, VCD ranked 1st (99.2) which meant that patients in VCD group had the best effect regarding mean IIEF scores within 3 months after RP (Figure 4A). No significant difference was observed between VCD group and V20DT group (MD: 5.44; 95\% CI: -0.81 to 11.69 ). Patients in 200AOD showed a significant improvement in mean IIEF scores when compared to $100 \mathrm{mg}$ avanafil on demand (100AOD) (MD: 1.60; 95\% CI: 1.46 to 1.74). The SUCRA ranking of PFMT has been greatly improved with the assistance of biofeedback (BF) or electrical stimulation (ES). The second group contained 301 patients with four treatments. The network meta-analysis indicated that VCD and $50 \mathrm{mg}$ /day sildenafil (VC50DS) showed superiority over $50 \mathrm{mg} /$ day sildenafil (50DS) (MD: 3.75; 95\% CI: 2.74-4.76) and intraurethral alprostadil 125-250 $\mu \mathrm{g}$ (MD: 3.05; 95\% CI: 0.38 to 5.72 ), respectively (Figure $4 F$ ). The SUCRA ranking showed that VC50DS ranked 1st and 50DS ranked the fourth (Figure 4B).

Thirteen studies with 1,052 participants involved 16 interventions and presented available results for $\geq 6$ months mean IIEF scores after RP. Eight of sixteen interventions were associated with significant improvement in mean IIEF scores when compared to placebo (Figure 5). The MDs for interventions related to significant improvement ranged from 1.6 (95\% CI: $1.25-1.95)$ for $80 \mathrm{mg} /$ day atorvastatin (80DA) to 14.22 (95\% CI: $9.04-19.40$ ) for V20DT. $10 \mathrm{mg} /$ day atorvastatin and $50 \mathrm{mg}$ sildenafil on demand (10DA50SOD), V20DT, VCD, $20 \mathrm{mg} /$ day tadalafil (20DT), 


\begin{tabular}{|c|c|c|c|c|c|c|c|c|c|c|c|c|}
\hline Study & Country & Participants (age" and number) & GS & $\mathrm{PSA}^{*}(\mathrm{ng} / \mathrm{mL})$ & DM & TPDC & SUA & CLS & NES & Intervention & Outcome measures $^{8}$ & LoE \\
\hline $\begin{array}{l}\text { Aydogdu et al. } \\
\text { [2011] }\end{array}$ & Turkey & $\begin{array}{l}\mathrm{N}=74(87.8 \% \text { completed study); } \\
\mathrm{IG}=32 ; 56.2 ; \\
\mathrm{CG}=33 ; 58.1\end{array}$ & $<8$ & $6.1(3.6-9.6)$ & $\begin{array}{l}\text { CG: } 4 / 33 \\
\text { IG: } 5 / 32\end{array}$ & $\begin{array}{l}\text { At baseline, } 3,6,12 \\
\text { months }\end{array}$ & RRP & $\leq \mathrm{Ic}$ & BNES & $\begin{array}{l}\text { IG: } 3 \text { days/week Tadalafil } 20 \mathrm{mg} / \text { day) for } 6 \text { months following the } \\
\text { removal of urethral catheter; } \\
\text { CG: no use of tadalafil }\end{array}$ & IIEF-EF; SEP-2; SEP-3 & $1 \mathrm{~b}$ \\
\hline $\begin{array}{l}\text { Montorsi et al. } \\
{[2008]}\end{array}$ & $\begin{array}{l}\text { Europe, Canada, South } \\
\text { Africa, and USA }\end{array}$ & $\begin{array}{l}\mathrm{N}=628(95.3 \% \text { completed the study); } \\
\mathrm{IG1}=207 ; 57.4 ; \\
\mathrm{IG} 2=204 ; 56.8 ; \\
\mathrm{CG}=206 ; 57.1\end{array}$ & $\leq 7$ & $<10$ & NS & $\begin{array}{l}\text { At } 0,1,3,6,9,10 \\
11,12,13 \text { months }\end{array}$ & NS & I-II & BNES & $\begin{array}{l}\text { IG1=9 months } 10 \mathrm{mg} \text { vardenafil nightly } \\
\text { (which could be decreased to } 5 \mathrm{mg} \text { if required); } \\
\text { IG2 }=9 \text { months flexible-dose (starting at } 10 \mathrm{mg} \text { with the option to } \\
\text { titrate to } 5 \mathrm{mg} \text { or } 20 \mathrm{mg} \text { ), on-demand vardenafil; } \\
\mathrm{CG}=9 \text { months placebo }\end{array}$ & IIEF-EF; SEP-2; SEP-3 & $1 \mathrm{~b}$ \\
\hline $\begin{array}{l}\text { Montorsi } \\
\text { et al. [2014] }\end{array}$ & $\begin{array}{l}\text { nine European countries } \\
\text { and Canada }\end{array}$ & $\begin{array}{l}\mathrm{N}=423(83 \% \text { completed the study); } \\
\text { IG1=139; } 58.6 \text { (5.07); } \\
\text { IG2=143;57.5 (5.91); } \\
\mathrm{CG}=141 ; 57.6(5.69)\end{array}$ & $\leq 7$ & $<10$ & None & At 0,9 months & $\begin{array}{l}\text { ORP; RARP; } \\
\quad \text { LRP }\end{array}$ & $|1 c-| \mid 2 c$ & BNES & $\begin{array}{l}\text { IG1 }=9 \text { months, } 5 \mathrm{mg} \text { tadalafil once daily; } \\
\text { IG2=9 months, } 20 \mathrm{mg} \text { tadalafil on demand; } \\
\mathrm{CG}=\text { placebo }\end{array}$ & IIEF-EF; SEP-3; penile length & $1 \mathrm{~b}$ \\
\hline $\begin{array}{l}\text { Padma-Nathan } \\
\text { et al. [2008] }\end{array}$ & $\begin{array}{l}\text { North America, France, } \\
\text { Belgium and Australia }\end{array}$ & $\begin{array}{l}\mathrm{N}=123(61.7 \% \text { completed the study); } \\
\mathrm{IG1}=23 ; 55[6] \\
\mathrm{IG} 2=28 ; 55[6] \\
\mathrm{CG}=25 ; 57[7]\end{array}$ & $<8$ & $<20 \mu \mathrm{g} / \mathrm{L}$ & NS & $\begin{array}{l}\text { At baseline, } \\
3,6,9 \text { months }\end{array}$ & RRP & I-II & BNES & $\begin{array}{l}\text { IG1=50 mg nightly sildenafil once daily; } \\
\text { IG2=100 mg nightly sildenafil once daily; } \\
\text { CG = placebo }\end{array}$ & IIEF-EF; RigiScan & $2 \mathrm{~b}$ \\
\hline $\begin{array}{l}\text { Pavlovich et al. } \\
\text { [2013] }\end{array}$ & USA & $\begin{array}{l}\mathrm{N}=100 \\
(100 \% \text { completed the study); } \\
\text { IG }=50 ; 54.3[42-63] ; \\
\mathrm{CG}=50 ; 53.6[40-64]\end{array}$ & $<8$ & NS & $1 \%$ & $\begin{array}{l}\text { At } 1,3,6,9,12,13 \\
\text { months }\end{array}$ & LRP; RARP & $|1 c-| 12 a$ & Yes & $\begin{array}{l}\mathrm{IG}=\text { nightly } 50 \mathrm{mg} \text { sildenafil for } 1 \text { year; } \\
\mathrm{CG}=\text { on demand } 50 \mathrm{mg} \text { sildenafil for } 1 \text { year } \\
\text { (maximum six tablets/month) }\end{array}$ & IIEF-EF; EPIC & $1 \mathrm{~b}$ \\
\hline $\begin{array}{l}\text { Bannowsky et al. } \\
\text { [2012] }\end{array}$ & I.Germany & $\begin{array}{l}\mathrm{N}=36(100 \% \text { completed the study); } \\
61.4[52-71] ; \text { IG1 }=12 ; \text { IG2 }=12 ; \mathrm{CG} \\
=12 ;\end{array}$ & $<7$ & $\leq 10$ & NS & $\begin{array}{l}\text { At baseline, 3, 6, and } \\
12 \text { months }\end{array}$ & RRP & I-II & UNES & $\begin{array}{l}\text { IG1 }=12 \text { months, } 5 \mathrm{mg} / \text { day vardenafil; } \\
\text { IG2 }=12 \text { months, } 10 \mathrm{mg} / \text { day vardenafil; } \\
\mathrm{CG}=12 \text { months, placebo }\end{array}$ & IIEF-5 & $1 \mathrm{~b}$ \\
\hline $\begin{array}{l}\text { Montorsi et al. } \\
\text { [2004] }\end{array}$ & $\begin{array}{l}\text { Canada, Germany, Italy, } \\
\text { The Netherlands, Spain, } \\
\text { UK and USA }\end{array}$ & $\begin{array}{l}\mathrm{N}=303 ;(78.2 \% \text { completed the study); } \\
\mathrm{IG}=201 ; 59.6(5.0) ; \\
\mathrm{CG}=102 ; 59.8(5.2)\end{array}$ & NS & NS & NS & At baseline, 3 months & RRP & $\leq$ III & BNES & $\begin{array}{l}\mathrm{IG}=20 \mathrm{mg} \text { tadalafil for } 12 \text { weeks; } \\
\mathrm{CT}=20 \mathrm{mg} \text { placebo }\end{array}$ & IIEF-EF; SEP-2; SEP-3; GAQ & $2 \mathrm{~b}$ \\
\hline $\begin{array}{l}\text { Mulhall et al. } \\
\text { [2013] }\end{array}$ & USA & $\begin{array}{l}\text { N=298 (84.6\% completed the study); } \\
\text { IG1=99; } 58.9(5.88) ; \\
\text { IG2=99; } 57.5(6.60) ; \\
\text { IG3 }=58.6(5.87)\end{array}$ & $\leq 7$ & NS & None & At $0,1,2,3$ months & $\begin{array}{l}\text { RRP; ORP; } \\
\text { LRP; RARP }\end{array}$ & $\leq 11$ & BNES & $\begin{array}{l}\text { IG1 }=100 \mathrm{mg} \text { avanafil; } \\
\text { IG2=200 mg avanafil; } \\
\text { CG = placebo; } \\
\text { Duration: } 3 \text { months }\end{array}$ & IIEF-EF; SEP-2; SEP-3 & $1 \mathrm{~b}$ \\
\hline $\begin{array}{l}\text { Raina et al. } \\
{[2006]}\end{array}$ & USA & $\begin{array}{l}N=109(87 \% \text { completed the study); } \\
58.2 ; I G=74 ; C G=35\end{array}$ & $\leq 6$ & $<10$ & NS & $\begin{array}{l}\text { At baseline, } 0,9 \\
\text { months }\end{array}$ & NS & I-II & $\begin{array}{l}\text { No } \\
\text { limitation }\end{array}$ & $\begin{array}{l}\text { IG: VCD use daily for } 9 \text { months; } \\
\text { CG: no treatment }\end{array}$ & IIEF-5; penile length and circumference & $1 \mathrm{~b}$ \\
\hline $\begin{array}{l}\text { Köhler et al. } \\
\text { [2007] }\end{array}$ & USA & $\begin{array}{l}\mathrm{N}=28(100 \% \text { completed the study); } \\
\mathrm{IG}=17 ; 58.2 ; \\
\mathrm{CG}=11 ; 60.5\end{array}$ & $\begin{array}{l}\text { IG: } 6.5 ; \\
\text { CG: } 6.7\end{array}$ & $\begin{array}{l}\text { IG: } 7.0 ; \\
\text { CG: } 5.5\end{array}$ & $\begin{array}{l}\text { IG: } 1 / 17 \\
\text { CG: } 2 / 11\end{array}$ & $\begin{array}{l}\text { At baseline, 1, 3, 6, 9, } \\
12 \text { months }\end{array}$ & RRP & I-II & $\begin{array}{l}\text { BNES; } \\
\text { UNES }\end{array}$ & $\begin{array}{l}\text { IG: early intervention ( } 1 \text { months after RP), } \\
10 \text { min/day VCD for } 5 \text { months; } \\
\text { CG: control group ( } 6 \text { months after RP) }\end{array}$ & $\begin{array}{l}\text { IIEF-5; penile flaccid length, stretched } \\
\text { length, prepubic fat pad, and midshaft } \\
\text { circumference }\end{array}$ & $1 \mathrm{~b}$ \\
\hline $\begin{array}{l}\text { Engel et al. } \\
\text { [2011] }\end{array}$ & USA & $\begin{array}{l}\mathrm{N}=23(87 \% \text { completed the study); } \\
\mathrm{IG}=13 \\
\mathrm{CG}=10\end{array}$ & NS & NS & None & At $3,6,9,12$ months & RARP & NS & BNES & $\begin{array}{l}\text { IG: } 20 \mathrm{mg} / \text { day tadalafil three times per week plus a VCD, } \\
10 \text { minutes unbanded per day for at least } 5 \text { days weekly; } \\
\text { CG: } 20 \mathrm{mg} / \text { day tadalafil three times per week }\end{array}$ & IIEF-5; penile erection hardness & $1 \mathrm{~b}$ \\
\hline Liu et al. [2016] & China & $\begin{array}{l}\mathrm{N}=64(100 \% \text { completed the study); } \\
\text { IG }=32 ; 57.6(4.1) ; \\
\mathrm{CG}=32 ; 56.9(4.5)\end{array}$ & NS & NS & NS & At 0,3 months & ORP & NS & $\begin{array}{c}\text { No } \\
\text { limitation }\end{array}$ & $\begin{array}{l}\text { IG: } 3 \text { months, } 50 \mathrm{mg} / \text { day sildenafil nightly and VCD; } \\
\text { CG: } 3 \text { months, } 50 \mathrm{mg} / \text { day sildenafil nightly }\end{array}$ & $\begin{array}{l}\text { IIEF-5; penile length and } \\
\text { circumference; erectile hardness }\end{array}$ & $1 \mathrm{~b}$ \\
\hline
\end{tabular}

Table 1 (continued) 


\begin{tabular}{|c|c|c|c|c|c|c|c|c|c|c|c|c|}
\hline Study & Country & Participants (age" and number) & GS & $\mathrm{PSA}^{*}(\mathrm{ng} / \mathrm{mL})$ & DM & TPDC & SUA & CLS & NES & Intervention & Outcome measures ${ }^{8}$ & LoE \\
\hline $\begin{array}{l}\text { Laurienzo et al. } \\
\text { [2018] }\end{array}$ & Brazil & $\begin{array}{l}\mathrm{N}=132(93.1 \% \text { completed the study); } \\
\text { IG1=41; } 58.5(5.4) ; \\
\text { IG2=42; } 58(5.7) ; \\
\mathrm{CG}=40 ; 57.3(6.5)\end{array}$ & NS & NS & NS & $\begin{array}{l}\text { At baseline, 1, 3, } 6 \\
\text { months }\end{array}$ & NS & $\|2 a-\||| 3 b$ & NS & $\begin{array}{l}\text { IG1: PFMT, in dorsal decubitus, with flexed lower limbs, perform } \\
\text { contraction of the pelvic floor, followed by relaxation; } \\
\text { IG2: PFMT and ES, twice a week for } 7 \text { weeks, totaling } 14 \text { sessions; } \\
\text { CG: no treatment }\end{array}$ & IIEF-5 & $1 \mathrm{~b}$ \\
\hline $\begin{array}{l}\text { Glazener } \\
\text { et al. [2011] }\end{array}$ & UK & $\begin{array}{l}\mathrm{N}=411(95.1 \% \text { completed the study); } \\
\mathrm{IG}=205 ; 62.4(5.8) ; \\
\mathrm{CG}=206 ; 62.3(5.6)\end{array}$ & NS & NS & NS & At $3,6,9,12$ months & $\begin{array}{l}\text { LRP; abdomi- } \\
\text { nal; perineal }\end{array}$ & NS & NS & $\begin{array}{l}\text { IG: therapist-guided PFMT, four one to one sessions, } 3 \text { months; } \\
\text { CG: no treatment }\end{array}$ & $\begin{array}{l}\text { Number of men unable to achieve } \\
\text { any erection } 12 \text { months after prostate } \\
\text { surgery }\end{array}$ & $1 \mathrm{~b}$ \\
\hline $\begin{array}{l}\text { Geraerts } \\
\text { et al. [2016] }\end{array}$ & Belgium & $\begin{array}{l}\mathrm{N}=33(100 \% \text { completed the study); } \\
\mathrm{IG}=16 ; 61.1 \\
\mathrm{CG}=17 ; 61.5\end{array}$ & NS & NS & NS & At 0,3 months & ORP; RARP & NS & $\begin{array}{l}\text { BNES; } \\
\text { UNES }\end{array}$ & $\begin{array}{l}\text { IG: therapist-guided PFMT and ES, during } 10 \text { min } \\
\text { (biphasic symmetric current (constant voltage), intensity as high as } \\
\text { possible, not painful, frequency: } 50 \mathrm{~Hz} \text { and pulse duration: } 600 \mu \mathrm{\mu s} \\
\text { CG: no treatment }\end{array}$ & IIEF-EF; VAS & $1 \mathrm{~b}$ \\
\hline Prota et al. [2012] & Brazil & $\begin{array}{l}\mathrm{N}=52(62.5 \% \text { completed the study); } \\
\mathrm{IG}=17 ; 62.4(6.4) ; \\
\mathrm{CG}=16 ; 64.0(8.0)\end{array}$ & NS & NS & $\begin{array}{l}\text { IG: } 17.6 \% \text {; } \\
\text { CG: } 12.5 \%\end{array}$ & $\begin{array}{l}\text { At } 1,3,6 \text { and } 12 \\
\text { months }\end{array}$ & RRP & $\|$ IIII & NS & $\begin{array}{l}\text { IG: PFMT and BF, once a week for } 12 \text { weeks after catheter removal } \\
\text { at postoperatively day 15; } \\
\text { CG: no treatment }\end{array}$ & IIEF-5 & $2 b$ \\
\hline $\begin{array}{l}\text { de Lira } \\
\text { et al. [2019] }\end{array}$ & Brazil & $\begin{array}{l}\mathrm{N}=31(100 \% \text { completed the study); } \\
\mathrm{IG}=16 ; 67.3(5.63) ; \\
\mathrm{CG}=15 ; 63.53(7.62)\end{array}$ & $\begin{array}{l}\text { No } \\
\text { limitation }\end{array}$ & $\begin{array}{l}\text { IG: } 9.20(4.65) ; \\
\text { CG: } 14.1 \text { (11.19) }\end{array}$ & NS & At baseline, 3 months & Open RRP & $\|2 c-1\| 3 b$ & NS & $\begin{array}{l}\text { IG: PFMT and BF, two pre-RP physical therapist-guided PFMT } \\
\text { sessions, including exercises and electromyographic biofeedback, } \\
\text { and verbal and written instructions to continue PFMT until RP, which } \\
\text { was then resumed after urethral catheter removal; } \\
\text { CG: only usual post-prostatectomy care }\end{array}$ & IIEF-5; ICIQ-SF & $1 \mathrm{~b}$ \\
\hline Oh et al. [2020] & Korea & $\begin{array}{l}\mathrm{N}=84(97.6 \% \text { completed the study); } \\
\mathrm{IG}=40 ; 67.5(6.9) ; \\
\mathrm{CG}=42 ; 65.9(6.8)\end{array}$ & $\begin{array}{c}\text { No } \\
\text { limitation }\end{array}$ & NS & $\begin{array}{l}\text { IG: } 5 / 40 ; \text {;G: } \\
\text { 9/42 }\end{array}$ & $\begin{array}{l}\text { At baseline, } 1,2,3 \\
\text { months }\end{array}$ & RARP & NS & $\begin{array}{l}\text { BNES; } \\
\text { UNES }\end{array}$ & $\begin{array}{l}\text { IG: PFMT and BF, (I) four times per day; (II) } 10 \text { minutes per session } \\
\text { of exercise; (III) a minimum of } 10 \text { seconds of tension duration with } \\
\text { maximal tension intensity; } \\
\text { CG: PFMT }\end{array}$ & IIEF-5 & $1 \mathrm{~b}$ \\
\hline $\begin{array}{l}\text { McCullough et } \\
\text { al. [2010] }\end{array}$ & USA & $\begin{array}{l}\mathrm{N}=212 \text { (73.5\% completed the study); } \\
\mathrm{IG}=97 ; 56.8(6.4) ; \\
\mathrm{CG}=59 ; 55.6(5.9)\end{array}$ & $>7$ & 20 & NS & $\begin{array}{l}\text { At baseline, } 0,1,3,6 \\
\quad 9,10,11 \text { months }\end{array}$ & ORP; RARP & NS & BNES & $\begin{array}{l}\text { IG: nightly intraurethral alprostadil, initially } 125 \mu \mathrm{gg} \text { and dose titrated } \\
250 \mu \mathrm{g} \text { at } 1 \text { month and maintained for } 8 \text { months; } \\
\text { CG: } 9 \text { months } 50 \mathrm{mg} \text { sildenafil nightly }\end{array}$ & IIEF-EF & $2 b$ \\
\hline $\begin{array}{l}\text { Mulhall et al. } \\
\text { [2018] }\end{array}$ & USA & $\begin{array}{l}\mathrm{N}=131(94.6 \% \text { completed the study); } \\
\mathrm{IG}=59 ; 55.1(6.2) ; \\
\mathrm{CG}=65 ; 54.1(6.2)\end{array}$ & NS & NS & None & $\begin{array}{l}\text { At } 1 \mathrm{w}, 3 \mathrm{w}, 5 \mathrm{w}, 9 \mathrm{w}, \\
3 \mathrm{~m}, 6 \mathrm{~m}, 12 \mathrm{~m}, 18 \mathrm{~m}\end{array}$ & ORP & I-II & BNES & $\begin{array}{l}\text { IG: tacrolimus } 2-3 \mathrm{mg} \text { daily for } 27 \text { weeks ( } 1 \text { week prior to and } 6 \\
\text { months after RP) and followed up for } 2 \text { years after RP; } \\
\text { CG: placebo }\end{array}$ & IIEF-EF & $1 \mathrm{~b}$ \\
\hline $\begin{array}{l}\text { Siltari et al. } \\
\text { [2019] }\end{array}$ & Finland; Denmark & $\begin{array}{l}\mathrm{N}=158(74.6 \% \text { completed the study); } \\
\text { IG }=60 ; 64(58.5-68) ; \\
\mathrm{CG}=58 ; 64[58-69]\end{array}$ & $\begin{array}{c}\text { No } \\
\text { limitation }\end{array}$ & $\begin{array}{c}\text { No } \\
\text { limitation }\end{array}$ & $\begin{array}{l}\text { IG: } 10 \% \text {; CG: } \\
\quad 12 \%\end{array}$ & $\begin{array}{l}\text { At baseline, 3, 6, 9, } \\
12 \text { months }\end{array}$ & NS & I-III & $\begin{array}{c}\text { No } \\
\text { limitation }\end{array}$ & $\begin{array}{l}\text { IG: } 80 \mathrm{mg} \text { atorvastatin daily from study inclusion to the day of } \\
\text { surgery; } \\
\text { CG: placebo }\end{array}$ & IIEF-5 & $2 \mathrm{~b}$ \\
\hline $\begin{array}{l}\text { Hong et al. } \\
\text { [2007] }\end{array}$ & Korea & $\begin{array}{l}\mathrm{N}=50(86 \% \text { completed the study); } \\
\mathrm{IG}=20 ; 61.3(4.3) ; \\
\mathrm{CG}=23 ; 60.6(2.3)\end{array}$ & $\begin{array}{l}\text { IG: } 6.2(0.7) ; \\
\text { CG: } 6.1(1.2)\end{array}$ & $\begin{array}{l}\text { IG: } 7.97(2.3) ; \\
\text { CG: } 7.44(4.5)\end{array}$ & None & $\begin{array}{l}\text { At baseline, } 0,6 \\
\text { months }\end{array}$ & RRP & $I-I I$ & BNES & $\begin{array}{l}\text { IG: } 10 \mathrm{mg} \text { atorvastatin daily from postoperative days } 1 \text { to } 90 \text { and on } \\
\text { demand } 50 \mathrm{mg} \text { sildenafil; } \\
\text { CG: on demand } 50 \mathrm{mg} \text { sildenafil }\end{array}$ & IIEF-5 & $1 \mathrm{~b}$ \\
\hline
\end{tabular}

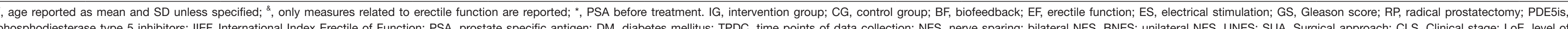

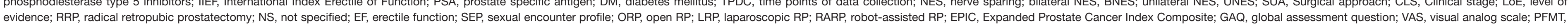
pelvic floor muscle training. 
Network plots

A

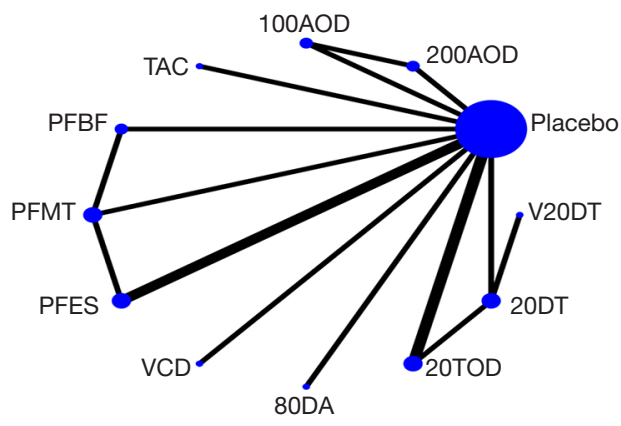

C

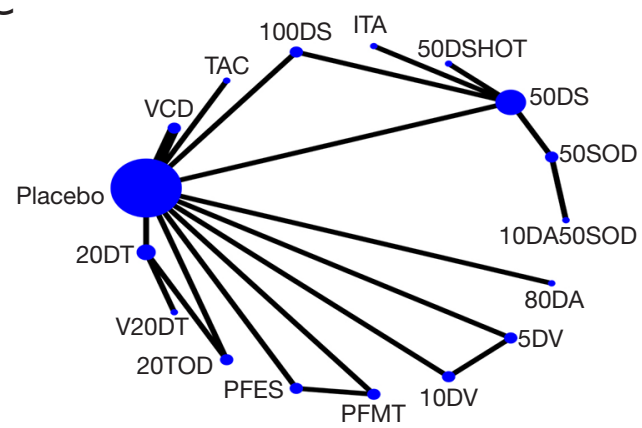

Funnel plots

E

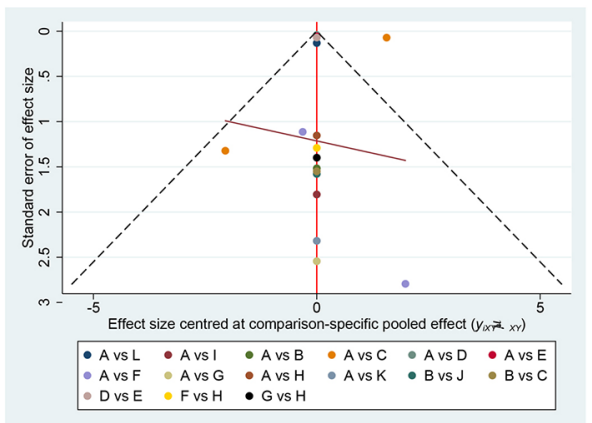

G

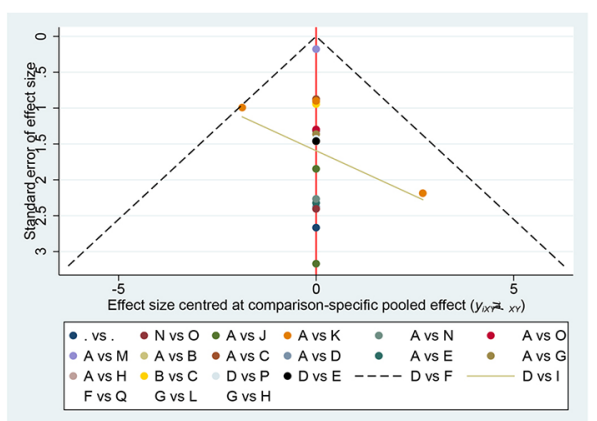

B
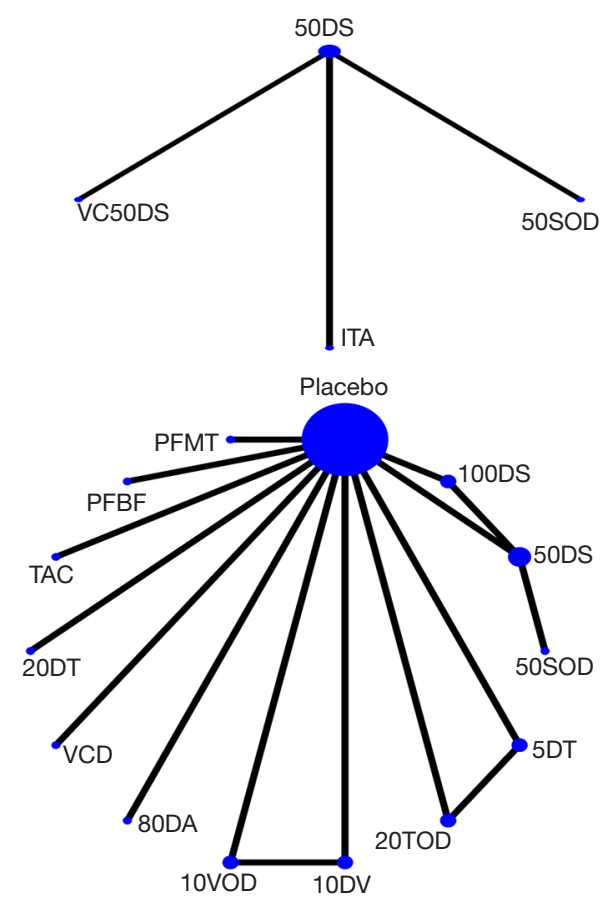

$\mathrm{F}$

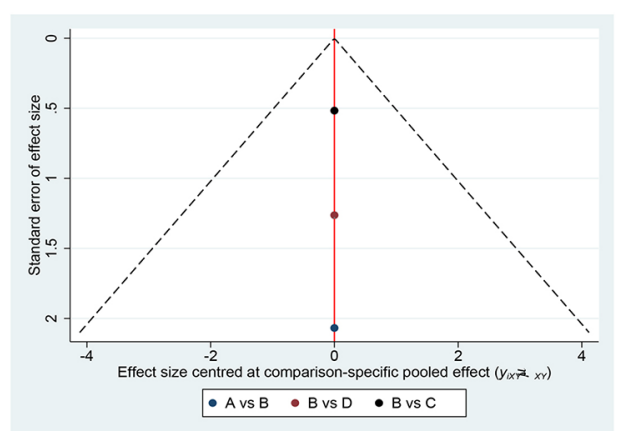

$\mathrm{H}$

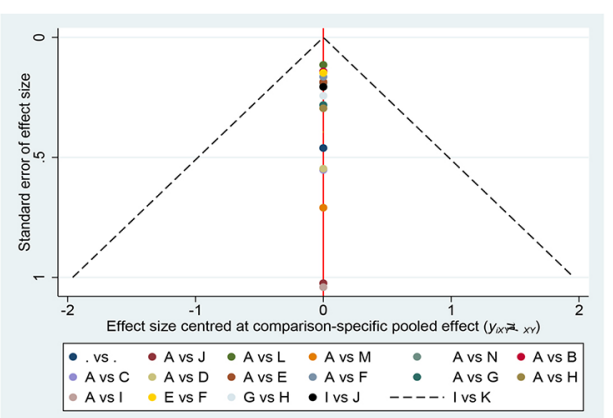

Figure 3 Network plots and funnel plots. Mean IIEF scores within 3 months after surgery: (A,E) and (B,F). Mean IIEF scores $\geq 6$ months after surgery: (C,G). The proportion of mean IIEF scores $\geq 22$ : (D,H). 
A

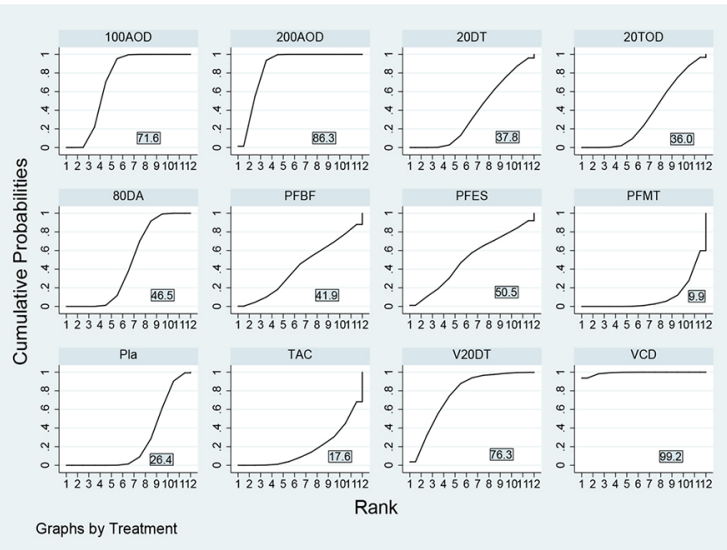

C
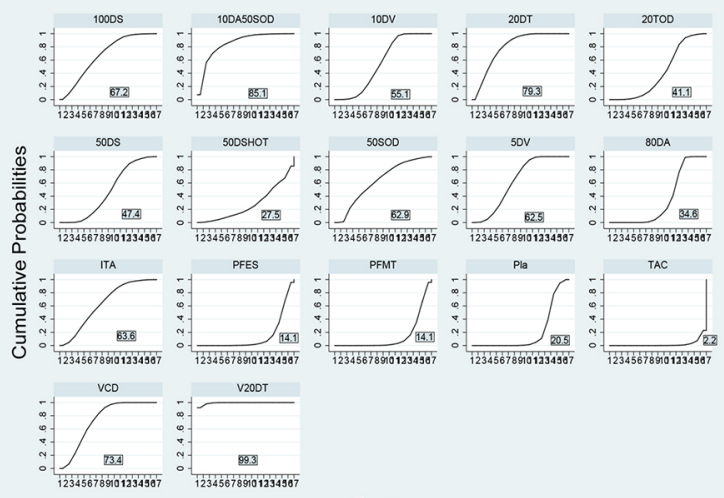

Graphs by Treatment

Rank

\section{E}

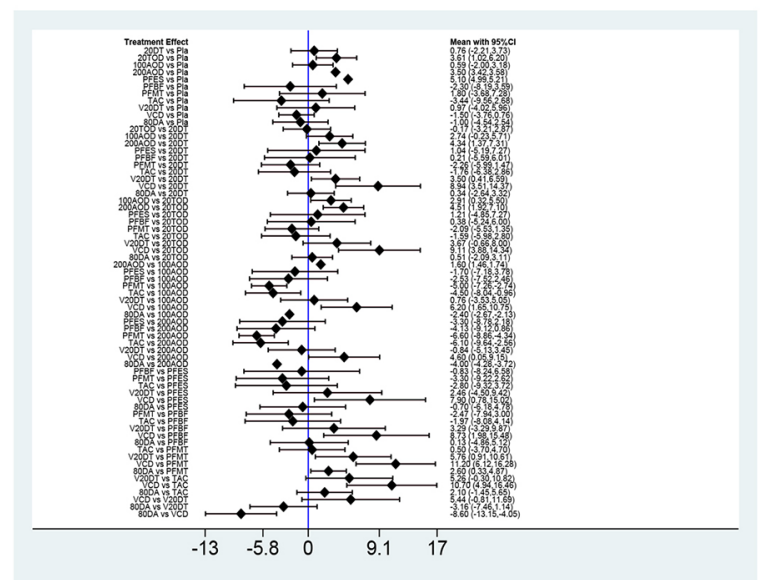

B

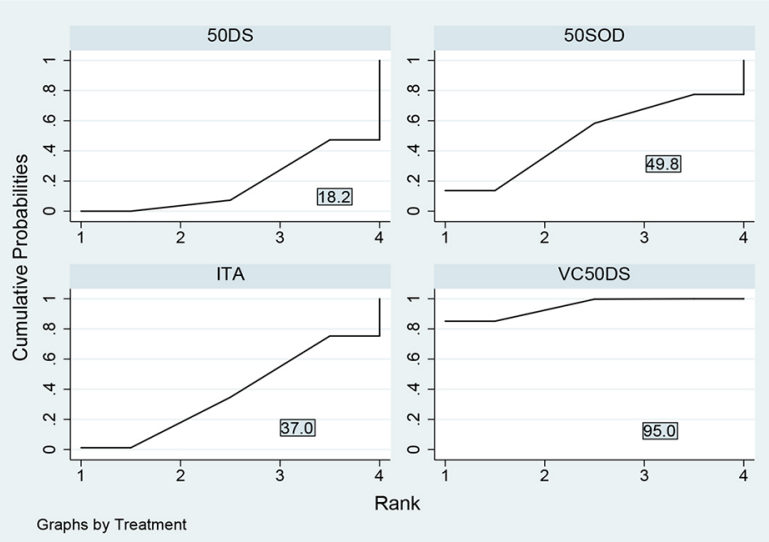

D
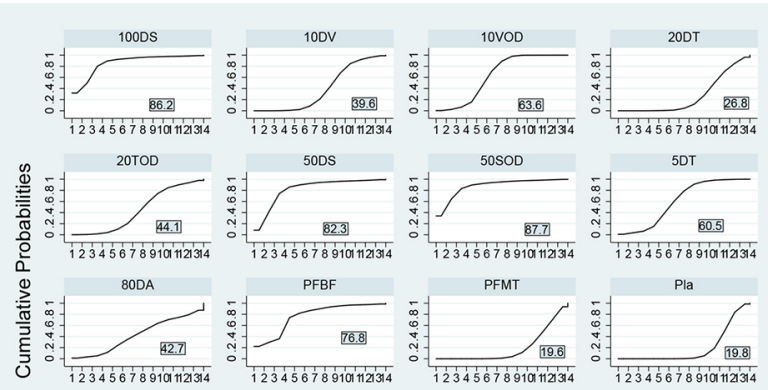

i2345678610101234
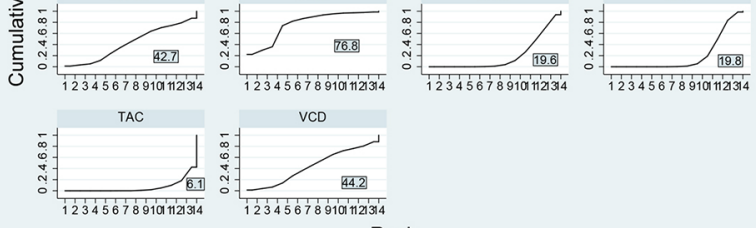

Graphs by Treatment

F

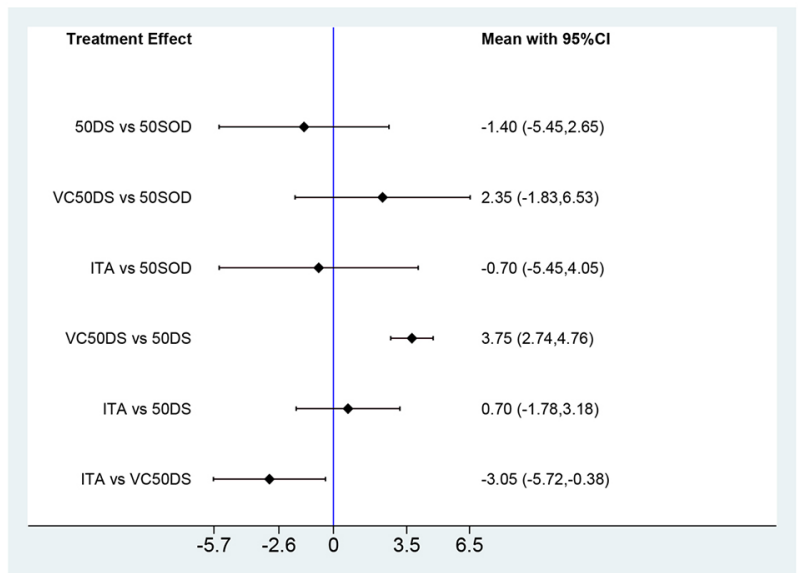

Figure 4 Surface under the curve cumulative probabilities (SUCRA) ranking and network results of mean IIEF scores within 3 months after surgery. Mean IIEF scores within 3 months after surgery: (A,E) and (B,F). Mean IIEF scores $\geq 6$ months after surgery: (C). The proportion of mean IIEF scores $\geq 22$ : (D). 


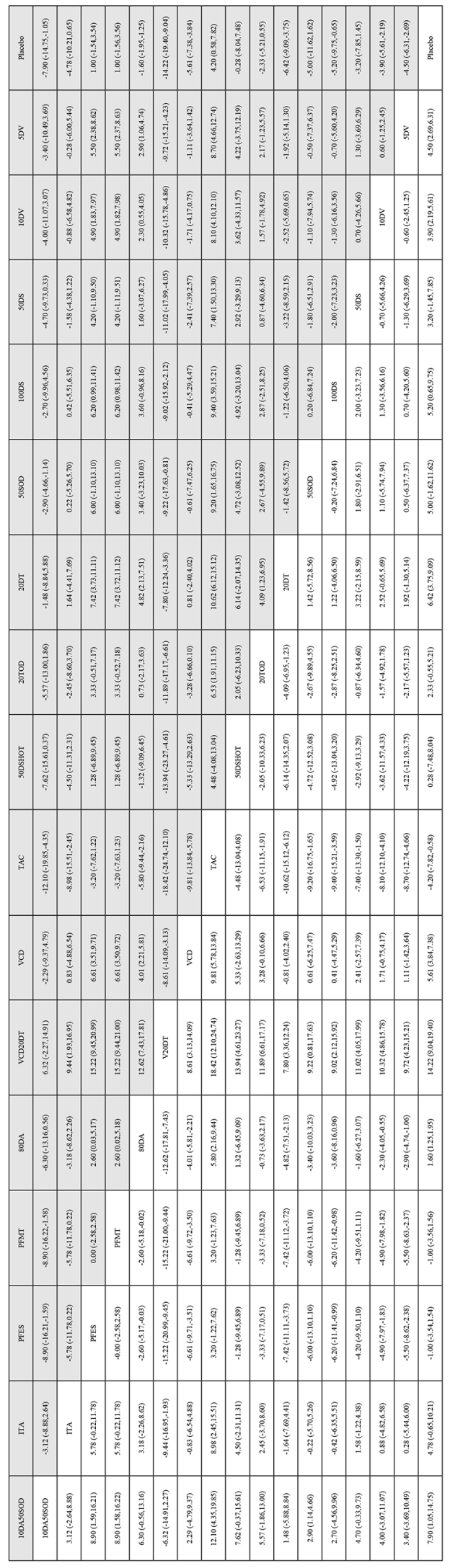

$10 \mathrm{mg}$ /day vardenafil (10DV), and $5 \mathrm{mg}$ /day vardenafil (5DV) improved mean IIEF scores significantly more than many other interventions (Figure 5). Moreover, V20DT showed significant superiority over the other interventions, which was consistent with the SUCRA ranking (Figure 4C).

\section{Network meta-analysis of the proportion of IIEF scores $\geq 22$}

Ten studies with 1,723 patients involved 13 interventions, and presented usable results for the proportion of IIEF scores $\geq 22$ greater than or equal to 6 months after RP. Of these interventions, only PFMT and BF (PFBF) were conducted as a combination therapy. Six of thirteen treatments had higher proportions of IIEF scores $\geq 22$ than tacrolimus, which was consistent with the SUCRA ranking (Figure 4D). Despite $50 \mathrm{mg}$ sildenafil on demand (50SOD) ranking 1st, most differences among these interventions were not significant (Figure 6). Notably, the results of network meta-analysis between the proportion of IIEF scores $\geq 22$ and mean IIEF scores $\geq 6$ months after RP were approximately congruent in terms of monotherapy.

\section{Consistency and convergence analysis}

No significant inconsistency was observed among the various interventions through node-splitting analysis, which meant the consistency model was reliable. No obvious loop inconsistency was detected by the test of loop inconsistency and the IF was relatively small (Figure 2). Besides, no significant publication bias was observed in the outcomes according to the funnel plot (Figure 3).

\section{Discussion}

Different from the other secondary impotency, postprostatectomy ED is mainly caused by neurovascular bundle injury. The erectile nerve plays an important role in the process of erection initiating and maintenance by activating the release of nitric oxides (NOs) and substantially increasing the prostaglandin E-1 (PGE-1) level (51-53). It has been demonstrated that nerve terminal released NOs have relaxant effects on the penis by increasing oxygenated blood flow to the erectile cavernous tissue and relaxing the smooth muscle fibers of the arteries and arterioles of the erectile tissue (54-58). This effect could be altered when the integrity of the nerve was damaged. Originated from the pelvic plexus, $\mathrm{CN}$ walks along the anterolateral side of the 


\begin{tabular}{|c|c|c|c|c|c|c|c|c|c|c|c|c|c|c|}
\hline $\begin{array}{l}\frac{8}{\frac{\pi}{\pi}} \\
\frac{\pi}{\pi}\end{array}$ & 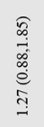 & $\begin{array}{l}\text { ह } \\
0 \\
0 \\
0 \\
0 \\
\text { ते } \\
0\end{array}$ & 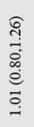 & 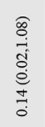 & 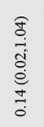 & 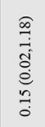 & 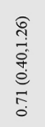 & 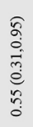 & 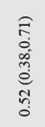 & \begin{tabular}{l} 
తa \\
\multirow{1}{*}{} \\
0 \\
0 \\
0 \\
0
\end{tabular} & 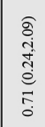 & 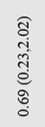 & 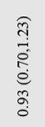 & 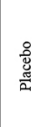 \\
\hline 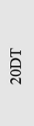 & 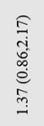 & 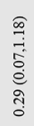 & 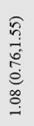 & 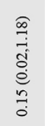 & 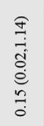 & $\begin{array}{l}\widehat{\widehat{T}} \\
\bar{d} \\
0 \\
0 \\
0 \\
0 \\
0\end{array}$ & 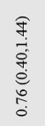 & $\begin{array}{l}\hat{\sigma} \\
\text { ì } \\
\hat{\tilde{O}} \\
\hat{\alpha} \\
\hat{o}\end{array}$ & $\begin{array}{l}\widehat{\sigma} \\
0 \\
0 \\
0 \\
0 \\
0 \\
0 \\
0\end{array}$ & 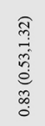 & 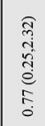 & 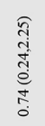 & 言 & $\begin{array}{l}\underset{g}{-} \\
\stackrel{0}{0} \\
\stackrel{0}{0} \\
\stackrel{0}{-}\end{array}$ \\
\hline ఫิ & 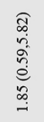 & $\begin{array}{l}\text { ठ } \\
\text { ป⿻ } \\
5 \\
0 \\
0 \\
0 \\
0\end{array}$ & 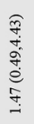 & 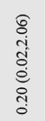 & 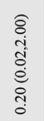 & 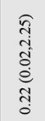 & 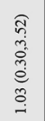 & 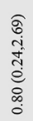 & 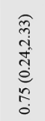 & $\begin{array}{l}\widehat{\sigma} \\
\hat{n} \\
0 \\
e \\
\tilde{0} \\
\stackrel{m}{=} \\
=\end{array}$ & 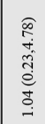 & ఫ్ర & 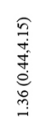 & 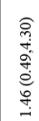 \\
\hline ఏ્ळ & 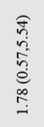 & 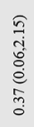 & 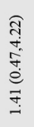 & 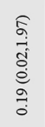 & 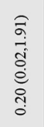 & 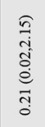 & 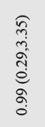 & 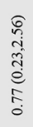 & 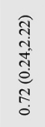 & 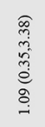 & 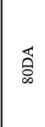 & 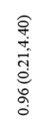 & $\begin{array}{l}\widehat{\sigma} \\
0 \\
\tilde{q} \\
\tilde{q} \\
0 \\
0 \\
0\end{array}$ & 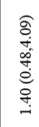 \\
\hline 㝑 & 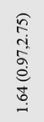 & 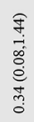 & 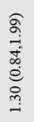 & 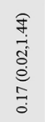 & 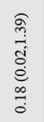 & 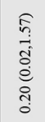 & 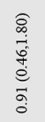 & 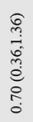 & $\begin{array}{l}\widehat{\alpha} \\
0 \\
0 \\
0 \\
0 \\
0 \\
0 \\
0\end{array}$ & 总 & 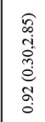 & 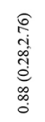 & 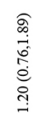 & 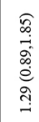 \\
\hline $\begin{array}{l}\text { ิ } \\
\text { ప્ }\end{array}$ & 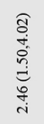 & 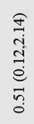 & 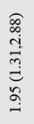 & 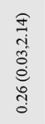 & 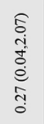 & 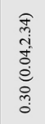 & $\begin{array}{l}\widehat{b} \\
0 \\
0 \\
0 \\
0 \\
0 \\
0 \\
-3\end{array}$ & 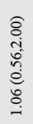 & $\begin{array}{l}\text { oิ } \\
\text { ؛े }\end{array}$ & 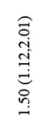 & $\begin{array}{l}\widehat{a} \\
+ \\
+ \\
0 \\
0 \\
0 \\
0 \\
0\end{array}$ & 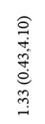 & 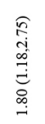 & 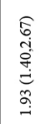 \\
\hline nิ & 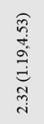 & $\begin{array}{l}\text { E } \\
\text { y. } \\
\overline{8} \\
\text { oे }\end{array}$ & 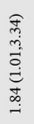 & 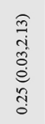 & 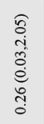 & 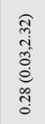 & 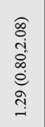 & 㞩 & 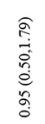 & 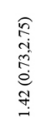 & 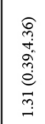 & 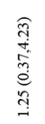 & 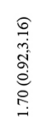 & 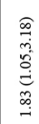 \\
\hline స్ & 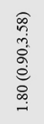 & 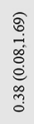 & 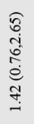 & $\begin{array}{l}\overline{8} \\
\text { - } \\
0 \\
0 \\
0 \\
0\end{array}$ & 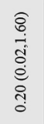 & 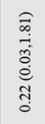 & 웜 & 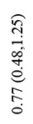 & 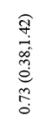 & 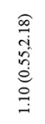 & $\begin{array}{l}\widehat{\gamma} \\
\dot{\gamma} \\
0 \\
0 \\
0 \\
0 \\
0 \\
-\end{array}$ & 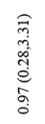 & 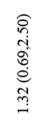 & 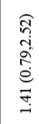 \\
\hline 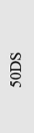 & 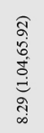 & 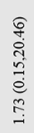 & 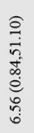 & 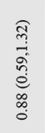 & 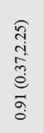 & 产 & 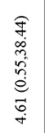 & 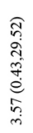 & 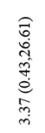 & 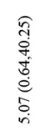 & $\begin{array}{l}6 \\
6 \\
0 \\
0 \\
0 \\
0 \\
0 \\
0 \\
0 \\
+ \\
+\end{array}$ & 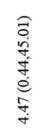 & $\begin{array}{l}E \\
\tilde{5} \\
+ \\
\hat{0} \\
0 \\
0 \\
0 \\
0\end{array}$ & $\begin{array}{l}\text { an } \\
0 \\
0 \\
0 \\
0 \\
0 \\
0 \\
0\end{array}$ \\
\hline $\begin{array}{l}\text { : } \\
\text { }\end{array}$ & $\begin{array}{l}\hat{\alpha} \\
\hat{0} \\
\infty \\
\vdots \\
\vdots \\
o \\
0\end{array}$ & $\begin{array}{l}\widehat{\sigma} \\
\text { ते } \\
\text { - } \\
0 \\
0 \\
0\end{array}$ & 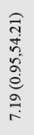 & 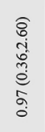 & $\begin{array}{l}0 \\
\text { ô }\end{array}$ & 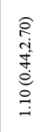 & 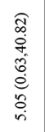 & 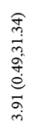 & 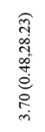 & 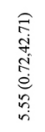 & 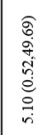 & 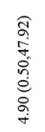 & $\begin{array}{l}\hat{f} \\
\stackrel{0}{0} \\
\infty \\
\infty \\
\infty \\
0 \\
0 \\
0 \\
0 \\
0\end{array}$ & 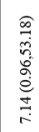 \\
\hline 宮 & 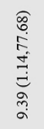 & 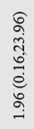 & 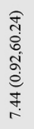 & 总 & 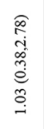 & 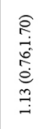 & 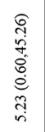 & 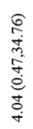 & 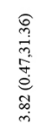 & 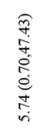 & 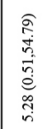 & 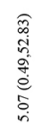 & 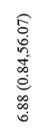 & $\begin{array}{l}\widehat{\hat{n}} \\
\text { dे } \\
\text { है } \\
\text { है }\end{array}$ \\
\hline 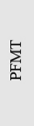 & 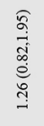 & 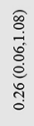 & 薏 & 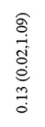 & 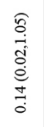 & 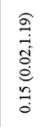 & $\begin{array}{l}\text { ले } \\
\stackrel{0}{0} \\
\hat{0} \\
\stackrel{0}{0} \\
\stackrel{0}{0} \\
0\end{array}$ & 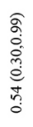 & 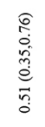 & $\begin{array}{l}\stackrel{0}{0} \\
\stackrel{1}{0} \\
0 \\
0 \\
\stackrel{0}{0} \\
0\end{array}$ & 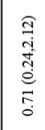 & 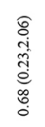 & 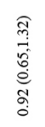 & $\begin{array}{l}\text { f } \\
0 \\
0 \\
0 \\
0 \\
0 \\
0\end{array}$ \\
\hline 呰 & 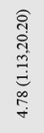 & 睘 & 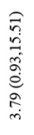 & 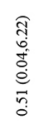 & $\begin{array}{l}\widehat{o} \\
0 \\
0 \\
0 \\
0 \\
0 \\
\tilde{n} \\
0\end{array}$ & $\begin{array}{l}\widehat{0} \\
0 \\
0 \\
0 \\
0 \\
0 \\
\infty \\
0 \\
0\end{array}$ & 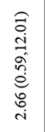 & 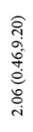 & 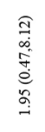 & 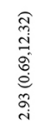 & 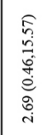 & 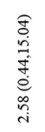 & 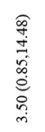 & 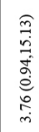 \\
\hline 巳 & 迎 & 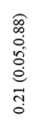 & 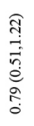 & $\begin{array}{l}\widehat{0} \\
0 \\
0 \\
0 \\
0 \\
0 \\
0\end{array}$ & 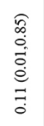 & 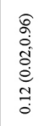 & 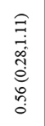 & 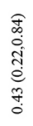 & 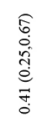 & $\begin{array}{l}\widehat{0} \\
\stackrel{0}{0} \\
\stackrel{0}{0} \\
\hat{0} \\
\stackrel{0}{0} \\
0\end{array}$ & $\begin{array}{l}\widehat{\widehat{x}} \\
0 \\
0 \\
0 \\
0 \\
0 \\
0 \\
0 \\
0\end{array}$ & 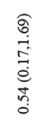 & $\begin{array}{l}\widehat{E} \\
\hat{0} \\
0 \\
0 \\
0 \\
0 \\
0 \\
0\end{array}$ & 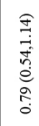 \\
\hline
\end{tabular}

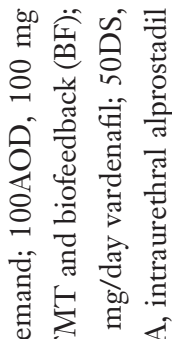

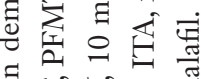

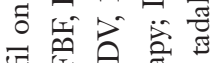

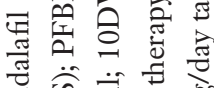
芯苛离

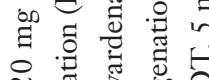

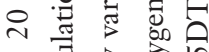

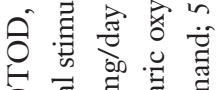
位.

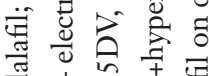
焉泀苚覀 过 $\sum_{i=0}$ के जि

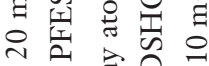
जे के

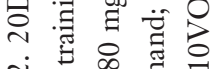
तิ

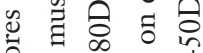
岙

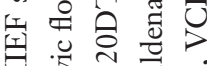

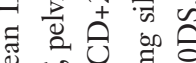

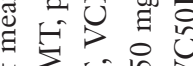
प $\sum_{i=1}$ in

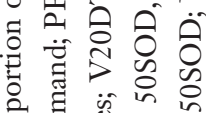

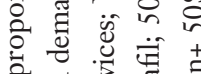
艺 놀 त ज्टु के

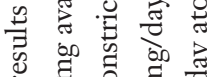
ङ .

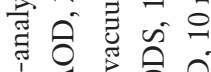

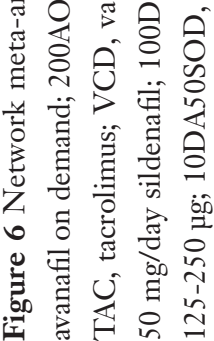


prostate (59). As the anatomical structure of the $\mathrm{CN}$ and prostate could be so tight, CNs injury is common during operation. Though more mini-invasive and precise surgical approaches like nsRARP have been widely recommended for its advantage in providing better visualization of the operative field for surgeons hopefully for protecting $\mathrm{CN}$ and a lower occurrence of RP induced ED was achieved, the morbidity of post-prostatectomy ED could still be high $(13,60)$. As is believed that not only by cutting, coagulation, traction, and compression of the pelvic tissues during operation could CNs injury be caused, postoperative local hypoxia and postoperative neuropraxia-related fibrotic and apoptotic changes in the erectile tissue were also responsible for post-prostatectomy $\mathrm{ED}(61,62)$. Despite the prominent understanding of the mechanism of post-prostatectomy ED, CNs injury is inevitable, even with the progress of surgical techniques. Thus, ED is a common sequela in patients after RP for clinically localized $\mathrm{PCa}$, with a negative impact on the quality of life and intimate relationship of patients and partners. Besides, the persistent lack of ED after neuropraxia can itself cause deleterious chain reactions that can negatively affect EF. Ligation of the accessory internal pudendal arteries and neuropraxia results in hypoxia and a lack of nocturnal erections. This results in corpus cavernosum fibrosis and the transformation of trabecular smooth muscle by collagen, which itself results in the loss of the venous occlusion mechanism required to maintain an erection (63). Nerve damage coupled with reduced arterial inflow may exacerbate hypoxia and ultimately lead to apoptosis (63). Therefore, the focus of improving ED after $\mathrm{RP}$ is to increase the amount of oxygen in the cavernous body and reduce tissue fibrosis and apoptosis.

Predictive factors for post-prostatectomy ED seem to be associated with age, preoperative baseline function, comorbidities index, an extension of the nerve-sparing procedure, surgery type (intrafascial, interfascial or extrafascial), surgical techniques (open, laparoscopic, RARP), operator's surgical experience, and the use of cautery-free dissection or the use of pinpointed lowenergy cauterization $(6,13)$. Although some patients might gradually recover 2 years or longer after surgery, only a few patients can return to their baseline EF (14). The concepts of penile rehabilitation were proposed to facilitate recovery of EF after RP as much as possible with any drug or device. Based on the current RCTs in our study, treatments currently available include PDE5is, PFMT, vacuum therapy, neuromodulatory therapy, statin therapy, intracorporeal injection therapy, hyperbaric oxygenation therapy, and combination therapies of these interventions. This network meta-analysis indicated that V20DT might have a better effect than other interventions within 1 year after surgery. However, the effectiveness of VCD may be affected by the equipment itself and the patients' experience. That's why VCD ranked 1st within 3 months after RP. PFMT might perform better with the guidance of $\mathrm{BF}$ and BS. Monotherapy appeared to have similar efficacy in terms of mean IIEF scores and proportion of patients return to baseline, and the effect of PDE5is did not seem to be affected by the patterns of administration (regular or on demand).

The goal of penile rehabilitation includes promoting early recovery of EF, reducing the loss of penis length and circumference, and improving overall sexual intercourse satisfaction. There is no definite conclusion regarding the timing of penile rehabilitation. Mulhall et al. (64) found patients in starting interventions within 6 months after surgery had a significantly higher mean IIEF-5 scores than those who starting interventions 6 months or longer after RP (22 vs. 16, $\mathrm{P}<0.001)$. Besides, stem cell therapy currently shows some promising results (65-67).

Despite ED after RP is a neurovascular injury event, psychological trauma may be a factor that cannot be ignored. Diagnosis of $\mathrm{PCa}$ is a life-changing event that can cause considerable psychological stress on patients and their sexual partners, and patients' anxiety can lead to ED before treatment after cancer diagnosis and before pathological results after prostate biopsy (68-70). The psychological effects before RP may have a substantial impact on preoperative and postoperative EF, exacerbating the adverse effects of surgery on EF (70).

The current trend in disease management is individualized treatment, and penile rehabilitation after RP is no exception. To our knowledge, this is the first study that synthetically commenced evaluating the efficacy of various interventions on penile rehabilitation for patients suffering from ED after RP. Despite comprehensive analysis being performed to make the summary results accurate and convincing, our study does have the following limitations. Firstly, limited RCTs might result in publication bias, and prevent us from reaching sufficient conclusions. Secondly, although considerable progress has been made in the study of EF rehabilitation after RP, there are still no conclusions in this field. Many studies lack the necessary data, such as using patient self-reported questionnaires, and there is significant heterogeneity, such as the definition of population, study design, and outcome measures. Besides, 
biological data such as penis scans or Doppler ultrasound are scarce. Finally, we were unlikely to further evaluate the long-term results of various treatments due to the lack of sufficient data in the included studies, which might make our analysis defective.

\section{Conclusions}

The combination therapy shows certain advantages over monotherapy, and we recommended the combination of VCD and PDE5 5 is to be considered in the clinical management of penile rehabilitation after RP. High-quality meta-analysis is still warranted based on well-designed RCTs, and other emerging therapies with promising results are also worth further exploring.

\section{Acknowledgments}

Funding: The study was supported by the 1.3 .5 project for disiplines of excellence, West China Hospital, Sichuan University (ZY2016104) and Department of Science and Technology of Sichuan Province (2020YFH0099). The funders had no role in study design, data collection or analysis, preparation of the manuscript, or the decision to publish.

\section{Footnote}

Reporting Checklist: The authors have completed the PRISMA reporting checklist. Available at http://dx.doi. org/10.21037/tau-20-892

Conflicts of Interest: All authors have completed the ICMJE uniform disclosure form (available at http://dx.doi. org/10.21037/tau-20-892). The authors have no conflicts of interest to declare.

Ethical Statement: The authors are accountable for all aspects of the work in ensuring that questions related to the accuracy or integrity of any part of the work are appropriately investigated and resolved.

Open Access Statement: This is an Open Access article distributed in accordance with the Creative Commons Attribution-NonCommercial-NoDerivs 4.0 International License (CC BY-NC-ND 4.0), which permits the noncommercial replication and distribution of the article with the strict proviso that no changes or edits are made and the original work is properly cited (including links to both the formal publication through the relevant DOI and the license). See: https://creativecommons.org/licenses/by-nc-nd/4.0/.

\section{References}

1. Siegel RL, Miller KD, Jemal A. Cancer statistics, 2015. CA Cancer J Clin 2015;65:5-29.

2. DeSantis CE, Lin CC, Mariotto AB, et al. Cancer treatment and survivorship statistics, 2014. CA Cancer J Clin 2014;64:252-71.

3. Brett AS, Ablin RJ. Prostate-cancer screening-what the U.S. Preventive Services Task Force left out. N Engl J Med 2011;365:1949-51.

4. Schroder FH. Stratifying risk-the U.S. Preventive Services Task Force and prostate-cancer screening. N Engl J Med 2011;365:1953-5.

5. Hamdy FC, Donovan JL, Lane JA, et al. 10-Year Outcomes after Monitoring, Surgery, or Radiotherapy for Localized Prostate Cancer. N Engl J Med 2016;375:1415-24.

6. Salonia A, Burnett AL, Graefen M, et al. Prevention and management of postprostatectomy sexual dysfunctions. Part.1: choosing the right patient at the right time for the right surgery. Eur Urol 2012;62:261-72.

7. Walz J, Burnett AL, Costello AJ, et al. A critical analysis of the current knowledge of surgical anatomy related to optimization of cancer control and preservation of continence and erection in candidates for radical prostatectomy. Eur Urol 2010;57:179-92.

8. Walz J, Epstein JI, Ganzer R, et al. A critical analysis of the current knowledge of surgical anatomy of the prostate related to optimisation of cancer control and preservation of continence and erection in candidates for radical prostatectomy: an update. Eur Urol 2016;70:301-11.

9. Asimakopoulos AD, Pereira Fraga CT, Annino F, et al. Randomized comparison between laparoscopic and robotassisted nerve-sparing radical prostatectomy. J Sex Med 2011;8:1503-12.

10. Park JW, Won Lee H, Kim W, et al. Comparative assessment of a single surgeon's series of laparoscopic radical prostatectomy: conventional versus robot-assisted. J Endourol 2011;25:597-602.

11. Cho JW, Kim TH, Sung GT. Laparoscopic radical prostatectomy versus robot-assisted laparoscopic radical prostatectomy: a single surgeon's experience. Korean J Urol 2009;50:1198-202.

12. Hakimi AA, Blitstein J, Feder M, et al. Direct comparison 
of surgical and functional outcomes of robotic-assisted versus pure laparoscopic radical prostatectomy: singlesurgeon experience. Urology 2009;73:119-23.

13. Ficarra V, Novara G, Ahlering TE, et al. Systematic review and meta-analysis of studies reporting potency rates after robot-assisted radical prostatectomy. Eur Urol 2012;62:418-30

14. Nelson CJ, Scardino PT, Eastham JA, et al. Back to baseline: erectile function recovery after radical prostatectomy from the patients' perspective. J Sex Med 2013;10:1636-43

15. Bratu O, Oprea I, Marcu D, et al. Erectile dysfunction post-radical prostatectomy - a challenge for both patient and physician. J Med Life 2017;10:13-8.

16. N. Mottet, P. Cornford, RCN. van den Bergh, et al. EAU Guidelines on prostate cancer. Access date, 2020. Available online: https://uroweb.org/guideline/prostatecancer/\#note_353

17. Burnett AL, Nehra A, Breau RH, et al. Erectile Dysfunction: AUA Guideline. J Urol 2018;200:633-41.

18. Liu C, Lopez DS, Chen M, et al. Penile Rehabilitation Therapy Following Radical Prostatectomy: A MetaAnalysis. J Sex Med 2017;14:1496-503.

19. Denison MR, Perlman S. Translation and processing of mouse hepatitis virus virion RNA in a cell-free system. J Virol 1986;60:12-8.

20. Kannan P, Winser SJ, Choi Ho L, et al. Effectiveness of physiotherapy interventions for improving erectile function and climacturia in men after prostatectomy: a systematic review and meta-analysis of randomized controlled trials. Clin Rehabil 2019;33:1298-309.

21. Lu X, Han H, Xing N, et al. Efficacy of sildenafil citrate in men with erectile dysfunction following bilateral nervesparing radical prostatectomy: systematic review and metaanalysis. Zhonghua Yi Xue Za Zhi 2015;95:2964-8.

22. Wang X, Wang X, Liu T, et al. Systematic review and meta-analysis of the use of phosphodiesterase type 5 inhibitors for treatment of erectile dysfunction following bilateral nerve-sparing radical prostatectomy. PLoS One 2014;9:e91327.

23. Moher D, Shamseer L, Clarke M, et al. Preferred reporting items for systematic review and meta-analysis protocols (PRISMA-P) 2015 statement. Syst Rev 2015;4:1.

24. Evidence-Based Cf. Oxford Centre for evidence-based medicine: levels of evidence, 2009. Available online: http:// www.cebm.net/oxford-centreevidence-based-medicinelevels-evidence-march-2009/

25. Hozo SP, Djulbegovic B, Hozo I. Estimating the mean and variance from the median, range, and the size of a sample. BMC Med Res Methodol 2005;5:13.

26. Yang L, Wang G, Du Y, et al. Remote ischemic preconditioning reduces cardiac troponin I release in cardiac surgery: a meta-analysis. J Cardiothorac Vasc Anesth 2014;28:682-9.

27. Aydogdu O, Gokce MI, Burgu B, et al. Tadalafil rehabilitation therapy preserves penile size after bilateral nerve sparing radical retropubic prostatectomy. Int Braz J Urol 2011;37:336-44.

28. Siltari A, Riikonen J, Fode M, et al. Effects of Preoperative Atorvastatin Treatment on Erectile Function After Radical Prostatectomy: Results from a Subgroup of ESTO1, a Randomized, Double-Blind, Placebo-Controlled Study. J Sex Med 2019;16:1597-605.

29. Canat L, Guner B, Gurbuz C, et al. Effects of three-timesper-week versus on-demand tadalafil treatment on erectile function and continence recovery following bilateral nerve sparing radical prostatectomy: results of a prospective, randomized, and single-center study. Kaohsiung J Med Sci 2015;31:90-5.

30. Montorsi F, Nathan HP, McCullough A, et al. Tadalafil in the treatment of erectile dysfunction following bilateral nerve sparing radical retropubic prostatectomy: a randomized, double-blind, placebo controlled trial. J Urol 2004;172:1036-41.

31. Mulhall JP, Burnett AL, Wang R, et al. A phase 3, placebo controlled study of the safety and efficacy of avanafil for the treatment of erectile dysfunction after nerve sparing radical prostatectomy. J Urol 2013;189:2229-36.

32. Bannowsky A, van Ahlen H, Loch T. Increasing the dose of vardenafil on a daily basis does not improve erectile function after unilateral nerve-sparing radical prostatectomy. J Sex Med 2012;9:1448-53.

33. Padma-Nathan H, McCullough AR, Levine LA, et al. Randomized, double-blind, placebo-controlled study of postoperative nightly sildenafil citrate for the prevention of erectile dysfunction after bilateral nerve-sparing radical prostatectomy. Int J Impot Res 2008;20:479-86.

34. Geraerts I, Van Poppel H, Devoogdt N, et al. Pelvic floor muscle training for erectile dysfunction and climacturia 1 year after nerve sparing radical prostatectomy: A randomized controlled trial. Int J Impot Res 2016;28:9-13.

35. de Lira GHS, Fornari A, Cardoso LF, et al. Effects of perioperative pelvic floor muscle training on early recovery of urinary continence and erectile function in men undergoing radical prostatectomy: a randomized clinical trial. Int Braz J Urol 2019;45:1196-203. 
36. Laurienzo CE, Magnabosco WJ, Jabur F, et al. Pelvic floor muscle training and electrical stimulation as rehabilitation after radical prostatectomy: A randomized controlled trial. J Phys Ther Sci 2018;30:825-31.

37. Oh JJ, Kim JK, Lee H, et al. Effect of personalized extracorporeal biofeedback device for pelvic floor muscle training on urinary incontinence after robot-assisted radical prostatectomy: A randomized controlled trial. Neurourol Urodyn 2020;39:674-81.

38. Mulhall JP, Klein EA, Slawin K, et al. A Randomized, Double-Blind, Placebo-Controlled Trial to Assess the Utility of Tacrolimus (FK506) for the Prevention of Erectile Dysfunction Following Bilateral Nerve-Sparing Radical Prostatectomy. J Sex Med 2018;15:1293-9.

39. Engel JD. Effect on sexual function of a vacuum erection device post-prostatectomy. Can J Urol 2011;18:5721-5.

40. Köhler TS, Pedro R, Hendlin K, et al. A pilot study on the early use of the vacuum erection device after radical retropubic prostatectomy. BJU Int 2007;100:858-62.

41. Pavlovich CP, Levinson AW, Su LM, et al. Nightly vs ondemand sildenafil for penile rehabilitation after minimally invasive nerve-sparing radical prostatectomy: results of a randomized double-blind trial with placebo. BJU Int 2013;112:844-51.

42. Liu, T, Chen, XG. Clinical diagnosis and treatment of erectile dysfunction after radical prostatectomy. Chinese Journal of Human Sexuality 2016;25:10-3.

43. McCullough AR, Hellstrom WG, Wang R, et al. Recovery of erectile function after nerve sparing radical prostatectomy and penile rehabilitation with nightly intraurethral alprostadil versus sildenafil citrate. J Urol 2010;183:2451-6.

44. Chiles KA, Staff I, Johnson-Arbor K, et al. A DoubleBlind, Randomized Trial on the Efficacy and Safety of Hyperbaric Oxygenation Therapy in the Preservation of Erectile Function after Radical Prostatectomy. J Urol 2018;199:805-11.

45. Raina R, Agarwal A, Ausmundson S, et al. Early use of vacuum constriction device following radical prostatectomy facilitates early sexual activity and potentially earlier return of erectile function. Int J Impot Res 2006;18:77-81.

46. Hong SK, Han BK, Jeong SJ, et al. Effect of statin therapy on early return of potency after nerve sparing radical retropubic prostatectomy. J Urol 2007;178:613-6.

47. Montorsi F, Brock G, Lee J, et al. Effect of nightly versus on-demand vardenafil on recovery of erectile function in men following bilateral nerve-sparing radical prostatectomy. Eur Urol 2008;54:924-31.
48. Montorsi F, Brock G, Stolzenburg JU, et al. Effects of tadalafil treatment on erectile function recovery following bilateral nerve-sparing radical prostatectomy: a randomised placebo-controlled study (REACTT). Eur Urol 2014;65:587-96.

49. Glazener C, Boachie C, Buckley B, et al. Urinary incontinence in men after formal one-to-one pelvicfloor muscle training following radical prostatectomy or transurethral resection of the prostate (MAPS): two parallel randomized controlled trials. Lancet 2011;378:328-37.

50. Prota C, Gomes CM, Ribeiro LHS, et al. Early postoperative pelvic-floor biofeedback improves erectile function in men undergoing radical prostatectomy: A prospective, randomized, controlled trial. Int J Impot Res 2012;24:174-8.

51. Kim N, Vardi Y, Padma-Nathan H, et al. Oxygen tension regulates the nitric oxide pathway. Physiological role in penile erection. J Clin Invest 1993;91:437-42.

52. Mulhall JP, Bivalacqua TJ, Becher EF. Standard operating procedure for the 52 . preservation of erectile function outcomes after radical prostatectomy. J Sex Med 2013;10:195-203.

53. Champion H, Abdel-Mageed A, Hellstrom W. Cavernous neurotomy causes hypoxia and fibrosis in rat corpus cavernosum. J Androl 2003;24:239-45.

54. Kim N, Azadzoi KM, Goldstein I, et al. A nitric oxidelike factor mediates nonadrenergic-noncholinergic neurogenic relaxation of penile corpus cavernosum smooth muscle. J Clin Invest 1991;88:112-8.

55. Ignarro LJ, Bush PA, Buga GM, et al. Nitric oxide and cyclic GMP formation upon electrical field stimulation cause relaxation of corpus cavernosum smooth muscle. Biochem Biophys Res Commun 1990;170:843-50.

56. Burnett AL, Lowenstein CJ, Bredt DS, et al. Nitric oxide: a physiologic mediator of penile erection. Science 1992;257:401-3.

57. Trigo-Rocha F, Hsu GL, Donatucci CF, et al. The role of cyclic adenosine monophosphate, cyclic guanosine monophosphate, endothelium and nonadrenergic, noncholinergic neurotransmission in canine penile erection. J Urol 1993;149:872-7.

58. Walsh PC. The discovery of the cavernous nerves and development of nerve sparing radical retropubic prostatectomy. J Urol 2007;177:1632-5.

59. Walz J, Graefen M, Huland H. Surgical anatomy of the prostate in the era of radical robotic prostatectomy. Curr Opin Urol 2011;21:173-8.

60. Gudmundsson J, Besenbacher S, Sulem P, et al. Genetic 
correction of PSA values using sequence variants associated with PSA levels. Sci Transl Med 2010;2:62ra92.

61. Saleh A, Abboudi H, Ghazal-Aswad MB, et al. Management of erectile dysfunction post-radical prostatectomy. Res Rep Urol 2015;7:19-33.

62. Moskovic DJ, Miles BJ, Lipshultz LI, et al. Emerging concepts in erectile preservation following radical prostatectomy: a guide for clinicians. Int J Impot Res 2011;23:181-92.

63. Wang R. Penile rehabilitation after radical prostatectomy: where do we stand and where are we going? J Sex Med 2007;4:1085-97.

64. Mulhall JP, Parker M, Waters BW, et al. The timing of penile rehabilitation after bilateral nerve-sparing radical prostatectomy affects the recovery of erectile function. BJU Int 2010;105:37-41.

65. Haahr MK, Harken Jensen C, Toyserkani NM, et al. A 12-Month Follow-up After a Single Intracavernous Injection of Autologous Adipose-Derived Regenerative Cells in Patients with Erectile Dysfunction Following Radical Prostatectomy: An Open-Label Phase I Clinical

Cite this article as: Feng D, Liu S, Yang Y, Bai Y, Li D, Han P, Wei W. Generating comprehensive comparative evidence on various interventions for penile rehabilitation in patients with erectile dysfunction after radical prostatectomy: a systematic review and network meta-analysis. Transl Androl Urol 2021;10(1):109-124. doi: 10.21037/tau-20-892
Trial. Urology 2018;121:203.e6-13.

66. Yiou R, Hamidou L, Birebent B, et al. Intracavernous Injections of Bone Marrow Mononucleated Cells for Postradical Prostatectomy Erectile Dysfunction: Final Results of the INSTIN Clinical Trial. Eur Urol Focus 2017;3:643-5.

67. Yiou R, Hamidou L, Birebent B, et al. Safety of Intracavernous Bone Marrow-Mononuclear Cells for Postradical Prostatectomy Erectile Dysfunction: An Open Dose-Escalation Pilot Study. Eur Urol 2016;69:988-91.

68. Lafaye A, Petit S, Richaud P, et al. Dyadic effects of coping strategies on emotional state and quality of life in prostate cancer patients and their spouses. Psychooncology 2014;23:797-803.

69. Zisman A, Leibovici D, Kleinmann J, et al. The impact of prostate biopsy on patient well-being: A prospective study of pain, anxiety and erectile dysfunction. J Urol 2001;165:445-54.

70. Xu P, Wang YH. Prevention and treatment of erectile dysfunction after prostatectomy: An update. Zhonghua Nan Ke Xue Za Zhi 2017;23:656-62. 